\title{
Aging and dietary restriction effects on ubiquitination, sumoylation, and the proteasome in the heart
}

\author{
Feng $\mathrm{Li}^{\mathrm{a}}$, Le Zhang ${ }^{\mathrm{b}}$, Jeffrey Craddock ${ }^{\mathrm{a}}{ }_{\text {Annadora J. Bruce-Keller }}^{\mathrm{b}}$, Kalavathi Dasuri ${ }^{\mathrm{b}}$, \\ AnhThao Nguyen ${ }^{b}$, and Jeffrey N. Keller $b$, \\ a Sanders-Brown Center on Aging, University of Kentucky, Lexington Kentucky, USA \\ b Pennington Biomedical Research Center/Louisiana State University System, Baton Rouge, LA, USA
}

\begin{abstract}
Dietary restriction (DR), in the absence of malnutrition, is the only intervention known to reliably increase average and maximal lifespan in a variety of organisms including mammals. Because the effects of DR on the heart are poorly understood, in the present study we examined the effects of DR on the ubiquitin-proteasome pathway (UPP) in the heart. In these studies we observed that DR significantly reduced age-related impairments in proteasome-mediated protein degradation, and reduced age-related increases in ubiquitinated, oxidized, and sumoylated protein in the heart. Interestingly, DR did not significantly increase the expression of $20 \mathrm{~S}$ proteasome subunits or the proteasome maturation factor (POMP-1). These data demonstrate for the first time the effects of aging and DR on proteasome biogenesis and sumoylation in the heart. Cumulatively, our data indicate that DR has many beneficial effects towards the UPP in the heart, and suggests that a preservation of the UPP may be a potential mechanism by which DR mediates beneficial effects on the cardiovascular system.
\end{abstract}

\section{Keywords}

Aging; Heart; Oxidative stress; Proteasome; Sumo; Ubiquitin

\section{Introduction}

Aging is associated with an alteration in steady state protein dynamics, including a dysfunction of both protein synthesis and protein degradation (Rattan, 1996, Szweda et al., 2003; Cuervo et al., 2005). Additionally, aging is associated with increased levels of proteins burdened with oxidative modifications (Grune, 2003; Szweda et al., 2003). Several studies have suggested that these age-related alterations to the proteome may play a role in promoting aging and agerelated disease in a variety of tissues (Rattan, 1996; Grune, 2000; Merker et al., 2001; Torres et al., 2003,2006; Ding et al., 2006). In particular studies have demonstrated a potential role for an age-related inhibition of a key intracellular protease, the proteasome, as a potential mediator of age-related pathogenesis in the heart (Keller et al., 2000a,b; Bulteau et al., 2002).

*Corresponding author: Dr Jeffrey N. Keller, Pennington Biomedical Research Center/LSU System, 6400 Perkins Road, Baton Rouge, LA 70808-4124 (P): 225-763-3190; (E): jeffrey.keller@pbrc.edu.

Publisher's Disclaimer: This is a PDF file of an unedited manuscript that has been accepted for publication. As a service to our customers we are providing this early version of the manuscript. The manuscript will undergo copyediting, typesetting, and review of the resulting proof before it is published in its final citable form. Please note that during the production process errors may be discovered which could affect the content, and all legal disclaimers that apply to the journal pertain. 
The ubiquitin proteasome pathway (UPP), is a proteolytic system which is responsible for a majority of intracellular protein degradation. The $20 \mathrm{~S}$ core proteasome is composed of alpha and beta subunits which together form a 28 subunit barrel shaped structure that is responsible for all proteasome-mediated protein degradation. Additional cap-like structures (devoid of proteolytic activity) can also bind to the $20 \mathrm{~S}$ proteasome to form a larger $26 \mathrm{~S}$ proteasome complex (Coux et al., 1996) The biogenesis of the 20S proteasome complex requires the protein proteoassemblin, which is also known as proteasome maturation protein (POMP1) (Ramos et al., 1998; Burri et al., 2000; Kruger et al., 2001).

A key aspect to UPP-mediated proteolysis is that proteins are selectively targeted for degradation, with two principle mechanisms of selectivity being the processes of ubiquitination and sumoylation (Melchior, 2000; Hermann et al., 2007). It is interesting to note that elevations in ubiquitinated and sumoylated protein are known to occur during aging of a variety of tissues (Grune, 2000; Szweda et al., 2003; Ding et al., 2006), with elevations in proteins possessing such post-translational modifications believed to represent malfunction of the UPP. However, it is important to point out that while impairments in peptidase activities of the proteasome have been demonstrated in the heart (Keller et al., 2000; Bulteau et al., 2002), to date it is unclear whether aging promotes an impairment in proteasome-mediated protein degradation.

Currently dietary restriction (DR) is the only intervention demonstrated to consistently and reliably increase mammalian lifespan. In addition to modulating lifespan, DR ameliorates the effects of aging on a variety of organ systems including hepatic, cardiovascular, immune, and central nervous system (Yu, 1996; Masuro, 2000; Pahlavani, 2004; Mattson and Wan, 2005). With regards to the cardiovascular system it is particularly important to note that DR reduces blood pressure, heart rate, atherosclerosis, and promotes an overall beneficial effect on the cardiovascular system (Nakano et al., 2001; Fontana et al., 2007; Minamiyama et al., 2007; Varady and Hellerstein, 2007). Currently is unclear which cellular and biochemical events are responsible for mediating the beneficial effects of DR in organs such as the heart. In the present study we demonstrate that aging and DR have multiple effects on the UPP in the heart, with the majority of these effects not described previously. Together, these data raise the possibility that alterations in the UPP within cardiomyocytes modulate heart physiology in response to aging and DR.

\section{Materials and Methods}

\subsection{Materials}

In this study, the POMP1/Ump1 (proteasome maturation protein) antibody (PW9715), 20S proteasome subunits $\alpha 1,2,3,5,6 \& 7(\mathrm{C} 2, \mathrm{C} 3, \mathrm{C} 8, \mathrm{C} 9$, iota and zeta) antibody (PW8195) and $20 \mathrm{~S}$ proteasome 'core' $(\beta)$ subunits antibody (PW8155) were purchase from Biomol International (Plymouth Meeting, PA, USA). Ub (P4D1) antibody (sc-8017) and SUMO-1 (D-11) antibody (sc-5308), were purchased from Santa Cruz Biotech, Inc. (Santa Cruz, CA, USA). The secondary antibodies peroxidase conjugated goat anti-rabbit $\operatorname{IgG}(\mathrm{H}+\mathrm{L})$ (111-035-003) and peroxidase conjugated goat anti-mouse $\operatorname{IgG}(\mathrm{H}+\mathrm{L})$ (115-035-003) were purchase from Jackson ImmunoResearch Lab, Inc. (West Grove, PA, USA). Protease Inhibitor Cocktail (P2714-1BTL) and all other reagents were obtained from Sigma (St. Louis, MO, USA).

\subsection{Animal studies}

Male Helicobacter-free F344/Brown Norway (F344 × BN F1) rats were obtained from the NIA Dietary Restriction (DR) colony. The hearts were manually dissected following euthanatization via $\mathrm{CO}_{2}$ overdose and subsequent decapitation. The rats in this study consisted of 6 three 
month-old ad libitum (AL), 6 twenty-five month old AL, and 6 twenty five month old DR rodents. These rodents were all utilized as outlined in IACUC approved protocols.

\subsection{Western blot analysis}

Western blot analysis was conducted to measure levels of POMP1, 20S proteasome $\alpha$ and $\beta$ subunits, and measure changes in protein ubiquitination and sumoylation cause by aging and DR in rat hearts. Rat hearts were homogenized in the presence of protease inhibitor cocktail, $15 \mu \mathrm{g}$ of each heart protein sample was separated by SDS-PAGE (BioRad, 7.5\% or 10-20\% precast gel) and transferred to nitrocellulose membrane (Whatman Schleicher \& Schuell Protran Nitrocellulose Transfer Membrane, Dassel, Germany). Membranes were then successively exposed to different primary and secondary antibodies before being developed using Pierce ECL Western Blotting Substrate (Pierce, Rockford, IL). The resulting bands were digitalized and quantified using NIH ImageJ software.

\subsection{Analysis of proteasome-mediated protein degradation}

Proteasome-mediated protein degradation was analyzed as described previously by our laboratory (Ceccarini et al., 2007). Briefly, $20 \mu \mathrm{g}$ lysates were prepared in $100 \mu \mathrm{l}$ of proteasome activity buffer (Keller et al., 2000a,b; Bulteau et al., 2002; Ceccarini et al., 2007) consisting of $10 \mathrm{mM}$ Tris- $\mathrm{HCl}$ ( $\mathrm{pH} 7.8), 0.5 \mathrm{mM}$ dithiothreitol, and $5 \mathrm{mM} \mathrm{MgCl}_{2}$. The samples were then incubated with $2 \mathrm{mg} / \mathrm{ml}$ of oxidized BSA, which was generated from the iron mediated oxidation of rhodamine labeled BSA (Molecular Probes, Eugene Oregon). Following a 30 minute oxidation, the BSA was dialyzed overnight and the presence of oxidation confirmed via analysis of protein carbonyls as described previously (Ceccarini et al., 2007). The oxidized BSA was incubated in the lysates for 1 hour, centrifugation, and ultimately the analysis of fluorescence. Proteasome-mediated protein degradation is reported as the percentage of the oxidized BSA which was degraded, which was determined by the change in fluorescence over time. The 26S proteasome activity (ATP stimulated, $1 \mathrm{mM} \mathrm{ATP)} \mathrm{and} \mathrm{20S} \mathrm{(SDS} \mathrm{stimulated,}$ $0.02 \%$ SDS) was measured in heart lysates using methodologies similar to what has been reported previously (Keller et al., 2000a,b; Kisselev et al., 2002). In order to confirm the specificity of the findings for proteasome activity all background protease activity in the heart lysate (not inhibited by $10 \mu \mathrm{M}$ MG132 pretreatment) was subtracted from the observed values.

\subsection{Analysis of Protein Oxidation}

The amount of protein oxidation was determined by analysis of protein carbonyls and 4hydroxynonenal (HNE) modified proteins as described previously (Keller et al., 2000; Bulteau et al., 2001; Ceccarini et al., 2007). Briefly, protein carbonyls were detected by derivatization of protein carbonyls, and subsequent detection of derivatized products using an antibody detection methodology (anti-dinitrophenylhydrazine: DNP antibody) available from a commercial source (Chemicon International, Temecula CA). HNE-modified proteins were detected using a commericially available antibody to HNE-lysine conjugates (Millipore, Temecula CA).

\section{Results}

\subsection{Effects of aging and DR on proteasome-mediated protein degradation and expression of 20S proteasome components}

Proteasome-mediated protein degradation was determined in lysates derived from the hearts of male 3 month-old AL, 25 month-old AL, and 25 month-old DR rodents obtained from the NIA rodent colony. Significant decreases in proteasome-mediated degradation of oxidized bovine serum albumin (osBSA) was observed in the hearts of aged AL rats (Figure 1). Interestingly, a nearly complete reversal of the age-related impairment in oxBSA degradation 
was observed in the hearts of aged DR rodents (Figure 1). In our next analysis we moved from analyzing proteasome-mediated protein degradation to measuring $26 \mathrm{~S}$ and $20 \mathrm{~S}$ proteasome peptidase activity. Analysis of $26 \mathrm{~S}$ proteasome activity in heart lysates revealed a significant, and surprising, increase in chymotrypsin-like activity in both aged and DR heart lysates (Figure 2). Analysis of $20 \mathrm{~S}$ proteasome activity in heart lysates revealed a significant decrease in chymotrypsin-like activity in the aging heart (Figure 2). While the trend for DR ameliorating age-related impairment in $26 \mathrm{~S}$ proteasome activity was observed (Figure 2), this observation was not statistically significant in the current sample set.

Because previous studies have demonstrated age-related impairments in proteasome function are related to elevations in oxidative modifications to the proteasome complexes (Keller et al., 2000a,b), we sought to elucidate if increased oxidative modification of the proteasome occurred in the heart tissues utilized for our aforementioned proteasome activity studies. In this analysis, following immunoprecipitation of the proteasome complexes, we observed that a significant amount of protein carbonyls were present in proteins of different molecular weights within the proteasome complexes all heart lysates examined (Figure 3), with no significant difference observed between the different experimental groups.

In order to elucidate if the aging- and DR-mediated alterations in proteasome-mediated protein degradation were associated with alteration in the expression of proteasome complexes we conducted Western blot analysis of 20S alpha and 20S beta subunits. In these analyses we observed that there was a decline in $20 \mathrm{~S}$ beta subunits in both aging AL and DR rodent hearts (Figure 4A), as compared to 3 month-old rats. In contrast to 20S beta subunit expression, 20S alpha subunits were observed to be elevated in both aged AL and DR rodents as compared to 3 month-old rat hearts (Figure 4B). Analysis is POMP-1 expression revealed no significant alteration in POMP-1 expression in either aged AL or DR rodents as compared to 3 month-old rat hearts (Figure 5), although a clear trend for decreased POMP-1 expression with both aging and DR was observed. For Western blot analysis of proteasome subunits and POMP-1 equal loading was confirmed by commassie blue staining (data not shown).

\subsection{Effects of aging and DR on the levels of ubiquitinated, sumoylated, and oxidized proteins in the heart}

In our next analysis we sought to examine the effects of aging and DR on the level of proteasome substrates (ubiquitinated-, sumoylated-, oxidized-proteins). In these analyses there was a significant elevation in the levels of ubiquitinated protein in the aged AL animals as compared to 3-month old rodents (Figure 6), with DR significantly attenuating the observed age-related increase in ubiquitinated protein levels (Figure 6). Interestingly, under our experimental conditions the increase in ubiquitinated protein was identified as likely being ubiquitin-loaded E1 and E2 ubiquitin ligases, as opposed to high-molecular weight smears of ubiquitin immunoreactivity. Similar to ubiquitination, aging was observed to elevate the levels of sumoylated protein in the aging heart (Figure 7), with DR again significantly reducing the agerelated elevation in sumoylated protein in the heart (Figure 7). For Western blot analysis of ubiquitinated and sumoylated proteins equal loading was confirmed by commassie blue staining (data not shown).

Because of the contribution of the proteasome to the degradation of oxidized proteins, we next sought to elucidate whether there was an alteration in oxidized protein levels in the heart tissue of aged AL and DR rodents. In this analysis we observed that while there was no significant alteration in 4-hydroxynonenal (HNE) modified proteins in any experimental group (Figure 8), there was observed to be a significant increase in protein carbonyls in the aged heart (Figure 8). DR was observed to significantly decrease age-related elevations in protein carbonyl levels within the majority of heart lysates examined (Figure 8). 


\section{Discussion}

The data in the present study demonstrate for the first time the effects of aging and DR on proteasome-mediated protein degradation in the heart. Additionally, our studies show that aging has different effects on the $26 \mathrm{~S}$ and $20 \mathrm{~S}$ proteasome peptidase activities in the heart. For example, $26 \mathrm{~S}$ proteasome activity is elevated in both aging AL and DR hearts, while $20 \mathrm{~S}$ proteasome activity is selectively inhibited in the hearts of aged AL rodents. This surprising divergence may have important implications for proteolysis in the heart where ubiquitinmediated proteolysis (26S proteasome) is likely able to be largely preserved as compared to $20 \mathrm{~S}$ proteasome activity (non-ubiquitin mediated proteolysis). In this scenario the aging heart would be expected to maintain the turnover of key 26S proteasome substrates (transcription factors, cyclins, etc.) but ultimately accumulate potentially toxic $20 \mathrm{~S}$ proteasome substrates such as oxidized proteins.

It appears that the basis for the decline in proteasome function in the aging heart may be mediated in part by a decline in the amount of $20 \mathrm{~S}$ beta proteasome subunits, which are the subunits responsible for the individual peptidase activities of the proteasome. Other studies have suggested that age-related impairments in $20 \mathrm{~S}$ proteasome function may be mediated by an increase in oxidative modifications to the proteasome complex (Grune, 2000; Szweda et al., 2003; Ding et al., 2006). However, in the present study we did not observe any significant alteration in oxidative modifications to the proteasome complexes present in heart lysates from either aged or DR rodents.

The ability of DR to preserve 20S proteasome peptidase activities, and proteasome-mediated degradation of oxidized proteins in the heart, appears not to be mediated by an increase in proteasome complexes. This is based on the fact that DR was not observed to increase the amount of 20S proteasome subunits or increase the expression of POMP1. It is interesting to note that in the present study POMP-1 was detected primarily as an apparent dimer, which has been observed previously (Zhang et al., 2007). It is possible that DR preserves proteasomemediated protein degradation in the aging heart via the suppression of the amount of oxidized proteins, which are not only substrates of the proteasome, but are capable of inhibiting proteasome activity (Bulteau et al., 2002). In the present study we observed reduced levels of protein carbonyls (Figure 7), but not HNE modified proteins, in the heart lysates of DR rodents which is consistent with DR-mediated decreases in the levels of protein carbonyls potentially contributing to the preservation of proteasome function.

Previous studies have strongly implicated a role for reduced level of reactive oxygen species (ROS) generation as the basis for DR mediated effects in tissues such as the heart (Yu, 1996; Minamiyama et al., 2007). These studies identified a key role for DR mediated suppression of mitochondrial ROS in mediating the beneficial effects of DR (Yu, 1996), with ROS known to be able to inhibit the proteasome in a variety of cell types (Grune, 2000; Szweda et al., 2003;Ding et al., 2006). Alternatively, recent studies have demonstrated that the heart possesses a number of proteasome subpopulations which presumably possess different compositions and different specific activities (Drews et al., 2007). DR may therefore promote the expression of proteasome complexes with higher specific activity, including $26 \mathrm{~S}$ proteasomes, which as a consequence aids in preserving proteasome-mediated protein degradation in the heart.

In the present study we observed that the age-related decline in proteasome-mediated protein degradation was associated with an elevation in both ubiquitinated and sumoylated protein. These data raise the possibility of age-related decreases in proteasome-mediated protein degradation potentially contribute to elevations in ubiquitinated and sumoylated protein in the aging heart. This model is further supported with the observation that the DR-mediated 
preservation in proteasome-mediated protein degradation is associated with a concomitant amelioration in ubiquitinated and sumoylated protein levels. Elevations in ubiquitinated and sumoylated protein are believed to represent the accumulation of aberrant proteins, which may directly or indirectly contribute to deleterious alterations in cell homeostasis. For example, the inability to tightly regulate cell signaling or transcription factors via proteolysis, or the inability to remove toxic misfolded or damaged proteins via degradation, could lead to pathological features in the heart as well as other tissues (Grune, 2000; Szweda et al., 2003). It is interesting to note that in our hands no significant elevation in high molecular weight smears of ubiquitinated protein were observed, and instead there appeared to be an elevation in what is likely E1 and/or E2 ubiquitin ligases. These data may indicate an alteration in the function of these two enzymes with age, whereby ubiquitin remains conjugated to these 2 enzymes with aging of the heart. Regardless, our data suggest that DR ameliorates age-related alterations in the proteome in part via the preservation of proteasome-mediated protein degradation.

The effect of proteasome inhibition on cardiomyocyte and heart function is complex. For example, studies 20 years ago identified that cardiac atrophy is associated with an increase in protein turnover (Samarel et al., 1987), as is the reversal of hypertrophy (Samarel et al., 1987). Recently, studies have demonstrated that application of proteasome inhibitors is sufficient to promote a number of pathological features in the heart including left ventricular atrophy (Stansfield et al., 2007). Additionally, studies have demonstrated that inhibition of the proteasome occurs in a number of pathological conditions including cardiac ischemiareperfusion injury (Bulteau et al., 2001). Taken together these data are consistent with proteasome inhibition being a deleterious and potentially pathological event in the heart. In contrast, proteasome inhibition has also been demonstrated to have beneficial effects on the heart in some instances, such as the ability of proteasome inhibition to induce beneficial preconditioning prior to cardiac ischemia-reperfusion injury (Patterson et al., 2007).

Additionally, some studies have demonstrated the ability of proteasome inhibitors to attenuate some forms of apoptosis (Ding et al., 2006; Patterson et al., 2007). It is therefore difficult to ascertain exactly what the effect of a DR-mediated preservation in proteasome function may be on the heart, although it is likely this preservation in proteasome function is an overall beneficial event to cardiomyocyte function, and may ultimately contribute to the beneficial effects of DR on the heart. Recent studies have demonstrated that proteasome inhibition is sufficient to impair protein synthesis, with feed-back between the proteasome and protein synthesis machinery contributing to the maintenance of cell homeostasis (Ding et al., 2006, 2007). Such studies raise the possibility that the preservation of proteasome function in the hearts of DR rodents is associated with a concomitant beneficial preservation in protein synthesis, which together contribute to the overall beneficial maintenance of the proteome, as compared to age-matched AL rodents.

It will be important in future studies to elaborate what alterations in the proteome are key for mediating the beneficial effects of DR in tissues such as the heart. Such efforts will almost certainly rely on accurately identifying crucial substrates of the proteasome, which also have altered turnover in the aging heart. This is necessary in large part because such proteins may serve as potentially important biomarkers of proteasome inhibition in the heart. Once such proteins are identified efforts can be made to identify which of these substrates has preserved expression-function in the hearts of DR rodents. Data from such experiments could thereby provide mechanistic insight as to how deleterious alterations in the proteome, mediated via age-related proteasome inhibition, may ultimately contribute to the effects of aging and DR on the heart.

\section{Acknowledgements}

This work was supported by grants from the National Institute of Aging (AG0257701, AG029885). 


\section{Abbreviations}

AL

ad libitum

DR

dietary restriction

NIA

National Institute of Aging

POMP

proteasome maturation protein

ROS

reactive oxygen species

Sumo

small ubiquitin-like modifier

Ub

ubiquitin

\section{References}

Asson-Batres MA, Zeng MS, Savchenko V, Aderoju A, McKanna J. Vitamin A deficiency leads to increased cell proliferation in olfactory epithelium of mature rats. J Neurobiol 2003;54:539-554 [PubMed: 12555267]

Bulteau AL, Lundberg KC, Humphries KM, Sadek HA, Szweda PA, Friguet B, Szweda LI. Oxidative modification and inactivation of the proteasome during coronary occlusion/reperfusion. J Biol Chem 2001;276:30057-30063. [PubMed: 11375979]

Bulteau AL, Szweda LI, Friguet B. Age-dependent declines in proteasome activity in the heart. Arch Biochem Biophys 2002;397:298-304. [PubMed: 11795886]

Burri L, Hockendorff J, Boehm U, Klamp T, Dohmen RJ, Levy F. Identification and characterization of a mammalian protein interacting with 20 S proteasome precursors. Proc Natl Acad Sci USA 2000;97:10348-10353. [PubMed: 10973495]

Ceccarni V, Ding Q, Keller JN. Oxidative inactivation of the proteasome in Alzheimer's disease. Free Radic Res 2007;41:673-680. [PubMed: 17516240]

Coux O, Tanaka K, Goldberg AL. Structure and functions of the $20 \mathrm{~S}$ and $26 \mathrm{~S}$ proteasomes. Annu Rev Biochem 1996;65:801-847. [PubMed: 8811196]

Cuervo AM, Bergamini E, Brunk UT, Droge W, Ffrench M, Terman A. Autophagy and aging: the importance of maintaining "clean" cells. Autophagy 2005;1:131-140. [PubMed: 16874025]

Ding Q, Ceccarini V, Keller JN. Interplay between protein synthesis and degradation in the CNS: physiological and pathological implications. Trends Neurosci 2007;30:31-36. [PubMed: 17126920]

Ding Q, Dimayuga E, Markesbery WR, Keller JN. Proteasome inhibition induces reversible impairments in protein synthesis. FASEB J 2006;20:1055-1063. [PubMed: 16770004]

Ding Q, Dimayuga ED, Keller JN. Proteasome regulation of oxidative stress in aging and age-related diseases of the CNS. Antioxid Redox Signal 2006;8:163-172. [PubMed: 16487050]

Drews O, Wildgruber R, Zong C, Sukop U, Nissum M, Weber G, Gomes AV, Ping P. Mammalian proteasome subpopulations with distinct molecular compositions and proteolytic activities. Mol Cell Proteomics 2007;6:2021-2031. [PubMed: 17660509]

Fontana L, Meyer TE, Klein S, Holloszy JO. Long-term calorie restriction is highly effective in reducing the risk for atherosclerosis in humans. Proc Natl Acad Sci USA 2004;101:6659-6663. [PubMed: 15096581] 
Grune T. Oxidative stress, aging and the proteasomal system. Biogerontology 2000;1:31-40. [PubMed: 11707918]

Herrmann J, Lerman LO, Lerman A. Ubiquitin and ubiquitin-like proteins in protein regulation. Circ Res 2007;100:1276-1291. [PubMed: 17495234]

Keller JN, Hanni KB, Markesbery WR. Possible involvement of proteasome inhibition in aging: implications for oxidative stress. Mech Ageing Dev 2000a;113:61-70. [PubMed: 10708250]

Keller JN, Huang FF, Markesbery WR. Decreased levels of proteasome activity and proteasome expression in aging spinal cord. Neuroscience 2000b;98:149-156. [PubMed: 10858621]

Kisselev AF, Kaganovich D, Goldberg AL. Binding of hydrophobic peptides to several non-catalytic sites promotes peptide hydrolysis by all active sites of $20 \mathrm{~S}$ proteasomes. Evidence for peptideinduced channel opening in the alpha-rings. J Biol Chem 2002;277:22260-22270. [PubMed: 11927581]

Kruger E, Kloetzel PM, Enenkel C. 20S proteasome biogenesis. Biochimie 2001;83:289-293. [PubMed: $11295488]$

Masoro EJ. Caloric restriction and aging: an update. Exp Gerontol 2000;35:299-305. [PubMed: 10832051]

Mattson MP, Wan R. Beneficial effects of intermittent fasting and caloric restriction on the cardiovascular and cerebrovascular systems. J Nutr Biochem 2005;16:129-137. [PubMed: 15741046]

Melchior F. SUMO--nonclassical ubiquitin. Annu Rev Cell Dev Biol 2000;16:591-626. [PubMed: 11031248]

Merker K, Stolzing A, Grune T. Proteolysis, caloric restriction and aging. Mech Ageing Dev 2001;122:595-615. [PubMed: 11322989]

Minamiyama Y, Bio Y, Takemura S, Takahashi Y, Kodai S, Mizuguchi S, Nishikawa Y, Suehiro S, Okada S. Calorie restriction improves cardiovascular riskfactors via reduction of mitochondrial reactive oxygen species in type II diabetic rats. J Pharmacol Exp Ther 2007;320:535-543. [PubMed: 17068205]

Nakano Y, Oshima T, Sasaki S, Higashi Y, Ozono R, Takenaka S, Miura F, Hirao H, Matsuura H, Chayama K, Kambe M. Calorie restriction reduced blood pressure in obesity hypertensives by improvement of autonomic nerve activity and insulin sensitivity. J Cardiovasc Pharmacol 2001;38:S69-S74. [PubMed: 11811363]

Pahlavani MA. Influence of caloric restriction on aging immune system. J Nutr Health Aging 2004;8:3847. [PubMed: 14730366]

Patterson C, Ike C, Willis PW, Stouffer GA, Willis MS. The bitter end: The ubiquitin-proteasome system and cardiac dysfunction. Circulation 2007;115:1456-1463. [PubMed: 17372187]

Ramos PC, Hockendorff J, Johnson ES, Varshavsky A, Dohmen RJ. Ump1p is required for proper maturation of the $20 \mathrm{~S}$ proteasome and becomes its substrate upon completion of the assembly. Cell 1998;92:489-499. [PubMed: 9491890]

Rattan SI. Synthesis, modifications, and turnover of proteins during aging. Exp Gerontol 1996;31:3347. [PubMed: 8706803]

Samarel AM, Parmacek MS, Magid NM, Decker RS, Lesch M. Protein synthesis and degradation during starvation induced cardiac atrophy in rabbits. Circ Res 1987;60:933-941. [PubMed: 3594760]

Stansfield WE, Tang R, Moss NC, Baldwin AS, Willis MS, Selzman CH. Proteasome inhibition promotes regression of left ventricle hypertrophy. Am J Physiol Heart Circ Physiol. 2007In press

Szweda PA, Camouse M, Lundberg KC, Oberley TD, Szweda LI. Aging, lipofuscin formation, and free radical-mediated inhibition of cellular proteolytic systems. Ageing Res Rev 2003;2:383-405. [PubMed: 14522242]

Torres C, Francis MK, Lorenzini A, Tresini M, Christofalo VJ. Metabolic stabilization of MAP kinase phosphatase-2 in senescence of human fibroblast. Exp Cell Res 2003;290:195-206. [PubMed: 14567979]

Torres C, Lewis L, Christofalo VJ. Proteasome inhibitors shorten replicative lifespan and induce a senescent-like phenotype of human fibroblast. J Cell Physiol 2006;207:845-853. [PubMed: 16523493]

Varady KA, Hellerstein MK. Alternate-day fasting and chronic disease prevention: a review of human and animal trials. Am J Clin Nutr 2007;86:7-13. [PubMed: 17616757] 
Yu BP. Aging and oxidative stress: modulation by dietary restriction. Free Radic Biol Med 1996;21:651668. [PubMed: 8891668]

Zhang L, Li F, Dimayuga E, Craddock J, Keller JN. Effects of aging and dietary restriction on ubiquitination, sumoylation, and proteasome in the spleen. FEBS Lett 2007;581:5543-5547.

[PubMed: 17991438] 


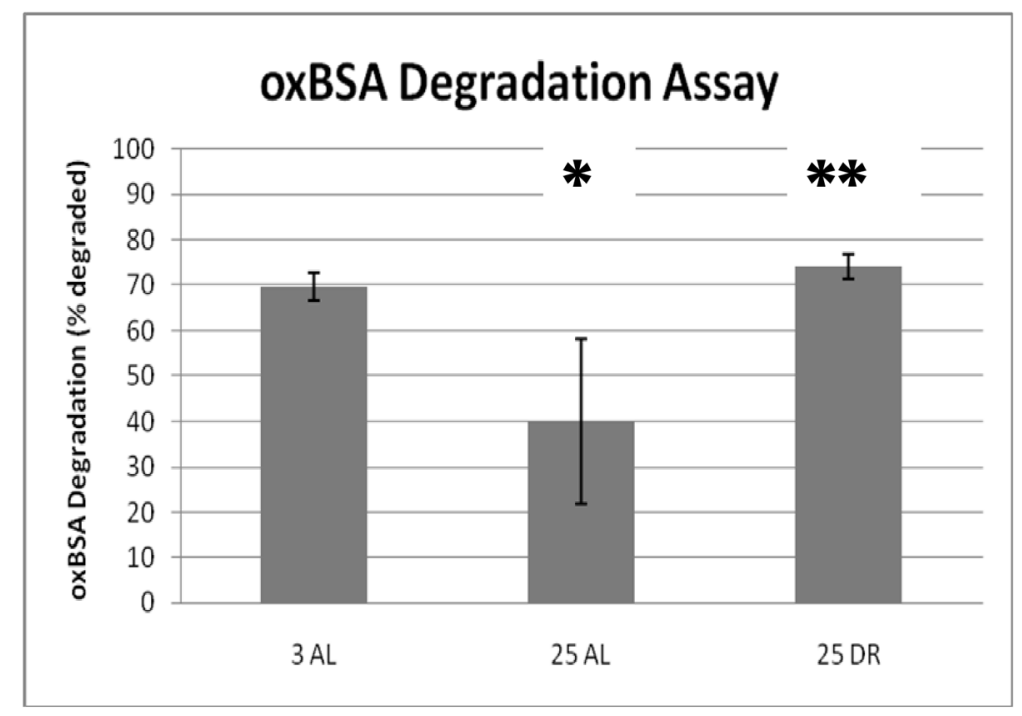

Figure 1. Effects of aging and dietary restriction on proteasome mediated protein degradation in the heart

The levels of proteasome mediated protein degradation were determined by measuring the amount of oxidized bovine serum albumin (oxBSA) in heart lysates from 3 month-old AL, 25 month-old AL, and 25 month-old DR rodents. The graphs represent the mean and S.E.M. of the degradation of rhodamine labeled oxBSA from 6 animals in each experimental group. *p $<0.05$ compared to 3 month old AL animals, **p< 0.05 compared to 25 month old AL rodents. 


\section{S Proteasome}

ATP-Associated Proteasome Activity

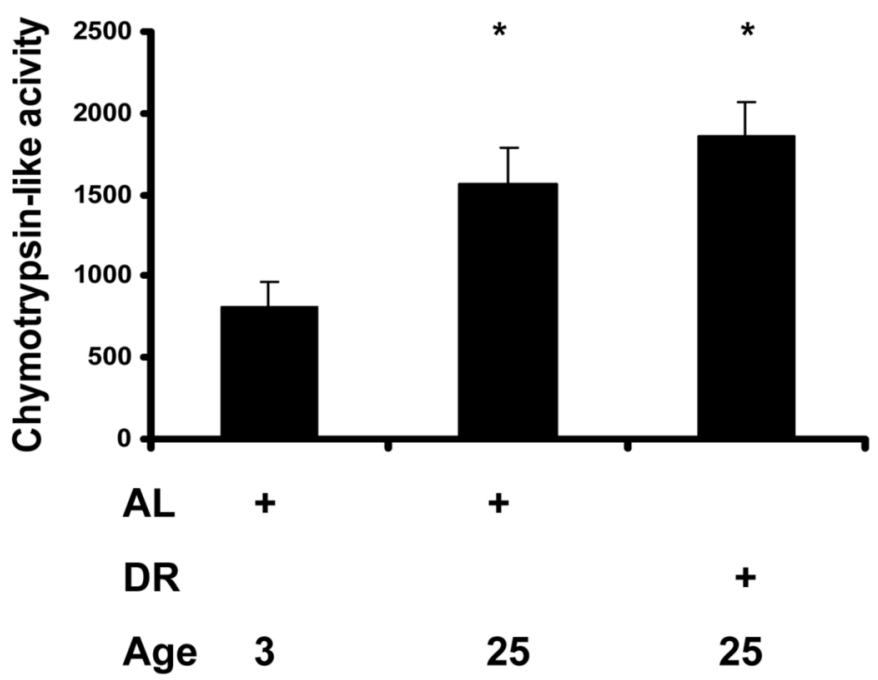

20S Proteasome

SDS-Associated Proteasome Activity

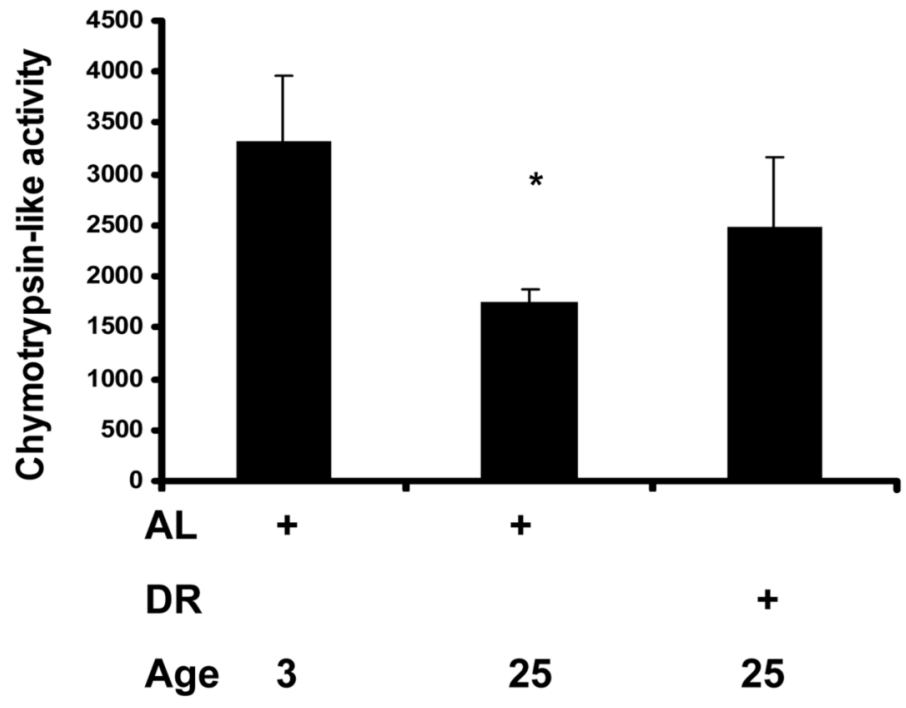

Figure 2. Analysis of heart lysate reveals an age-related impairment in 20S, and age-related increase in $26 \mathrm{~S}$, proteasome activity

Analysis of $26 \mathrm{~S}$ proteasome activity in heart lysates (ATP stimulated) revealed a significant elevation in chymotrypsin-like activity in both aged and DR heart lysates. Analysis of 20S proteasome activity (SDS stimulated) revealed an age-related impairment in chymotrypsinlike activity in 25-month old tissues relative to heart lysates from 3-month old animals. The specificity for proteasome activity in heart lysate studies was determined by subtracting the background protease activity (chymotrypsin-like activity not inhibited by lysate pretreatment with $10 \mu \mathrm{M}$ MG132). *p < 0.05 compared to 3 month old AL animals. 


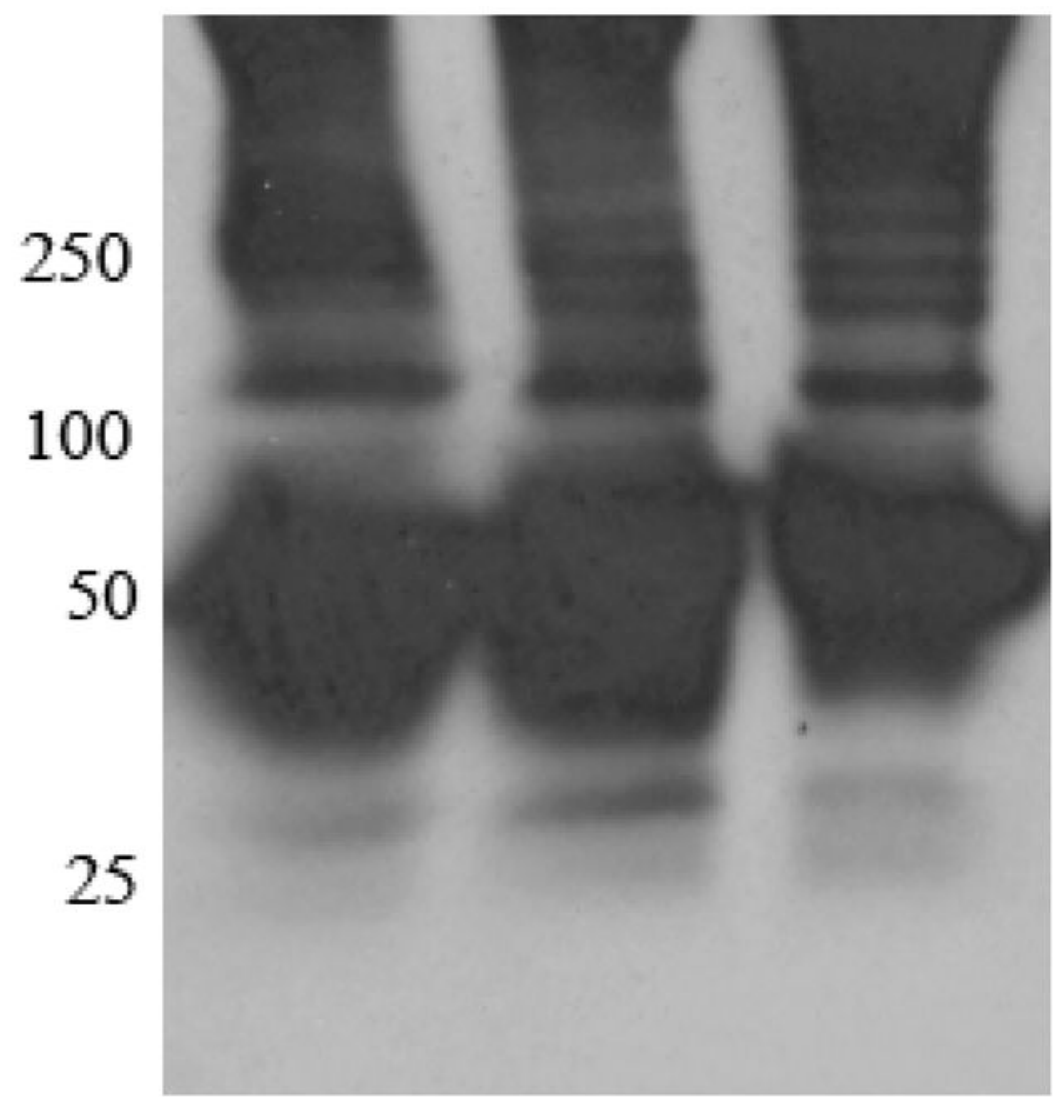

AL

DR

\section{AGE}

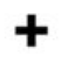

$+$

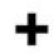

3

25

Figure 3. Oxidative modification to the proteasome in the heart does not appear to be modulated by aging or DR

Immunoprecipitation of the proteasome was conducted in heart lysates from 3 month-old AL, 25 month-old AL, and 25 month-old DR rodents. The resulting immunoprecipitate was analyzed for protein carbonyls by derivatization and subsequent detection of the derivatized products using an anti- DNP antibody based detection system. Results are representative of results from 6 separate experiments. 
A

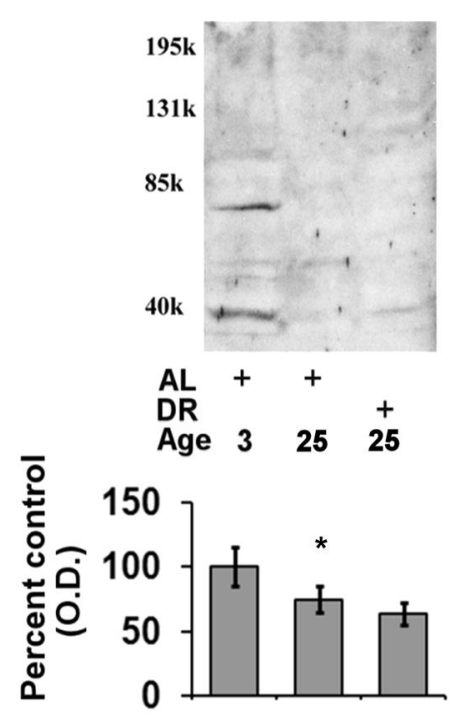

B

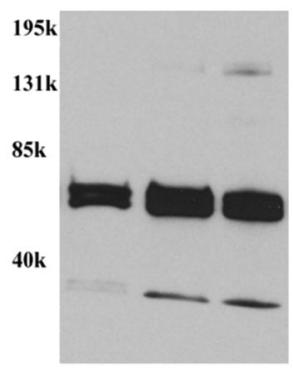

$\mathrm{AL}+\quad+$

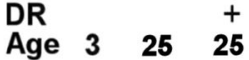

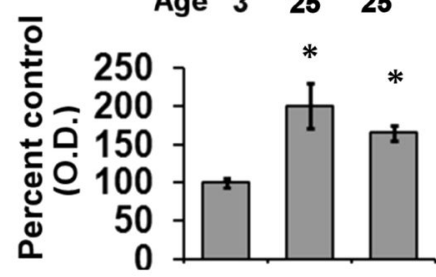

Figure 4. Effects of aging and dietary restriction on the levels of $20 \mathrm{~S}$ alpha and $20 \mathrm{~S}$ beta subunits in the heart

The level of 20S beta subunits (A) and 20S alpha subunits (B) were analyzed in the hearts of 3 month-old AL, 25 month-old AL, and 25 month-old DR rodents. A representative blot for the analyses is provided, with the molecular weight markers provided on left hand margin of the representative blot. The graph represent the mean and S.E.M. of the optical density of the immunoreactivity from 6 animals in each experimental group. *p $<0.05$ compared to 3 month old AL animals. Equal loading was confirmed by commassie staining, data not shown. 


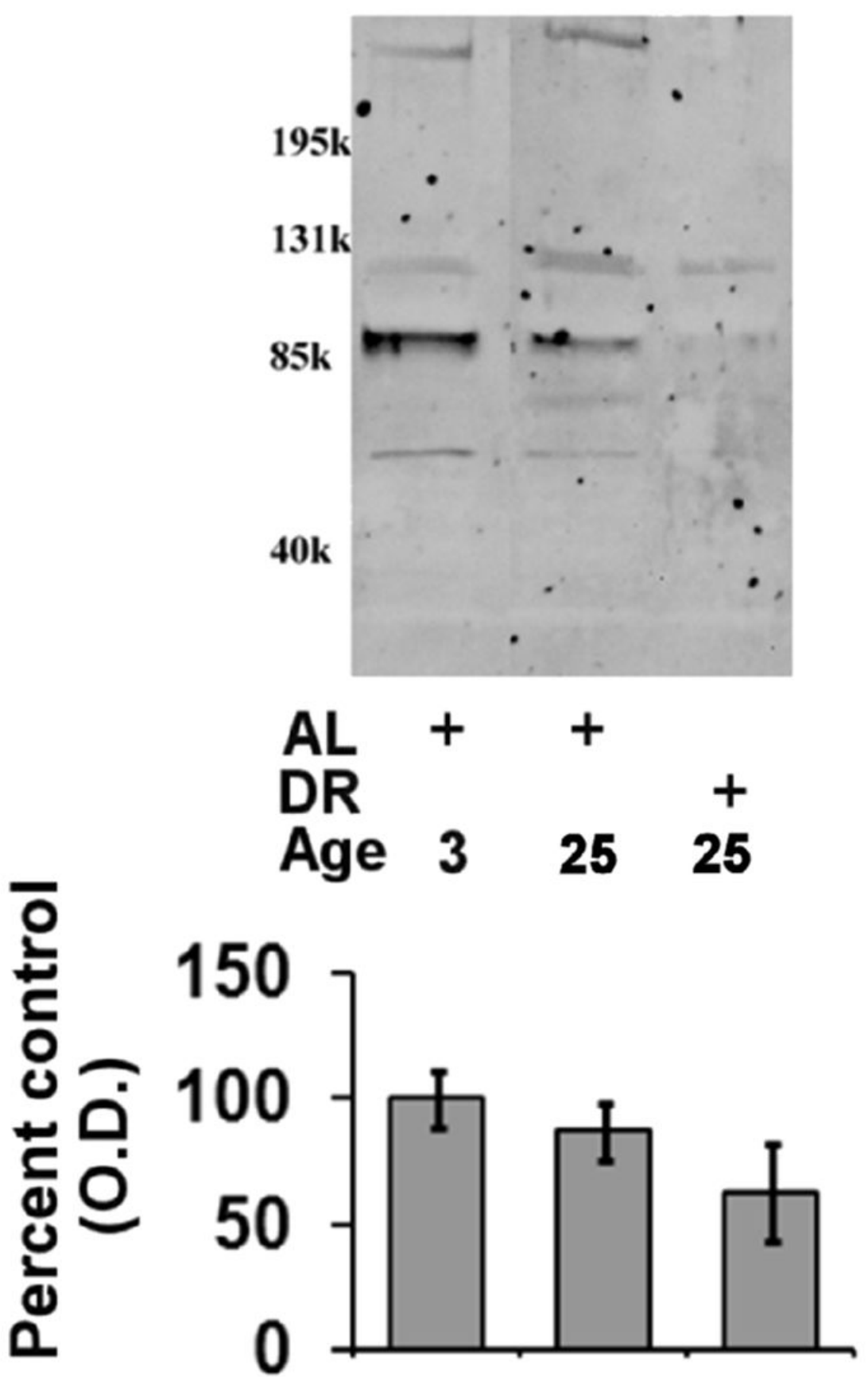

Figure 5. Effects of aging and dietary restriction on the levels of POMP-1 in the heart The level of POMP-1 were analyzed in the hearts of 3 month-old AL, 25 month-old AL, and 25 month-old DR rodents. A representative blot for the analyses is provided, with the molecular weight markers provided on left hand margin of the representative blot. The graph represent the mean and S.E.M. of the optical density of the immunoreactivity from 6 animals in each experimental group. Equal loading was confirmed by commassie staining, data not shown. 


\section{5k}

$131 k$

$85 k$
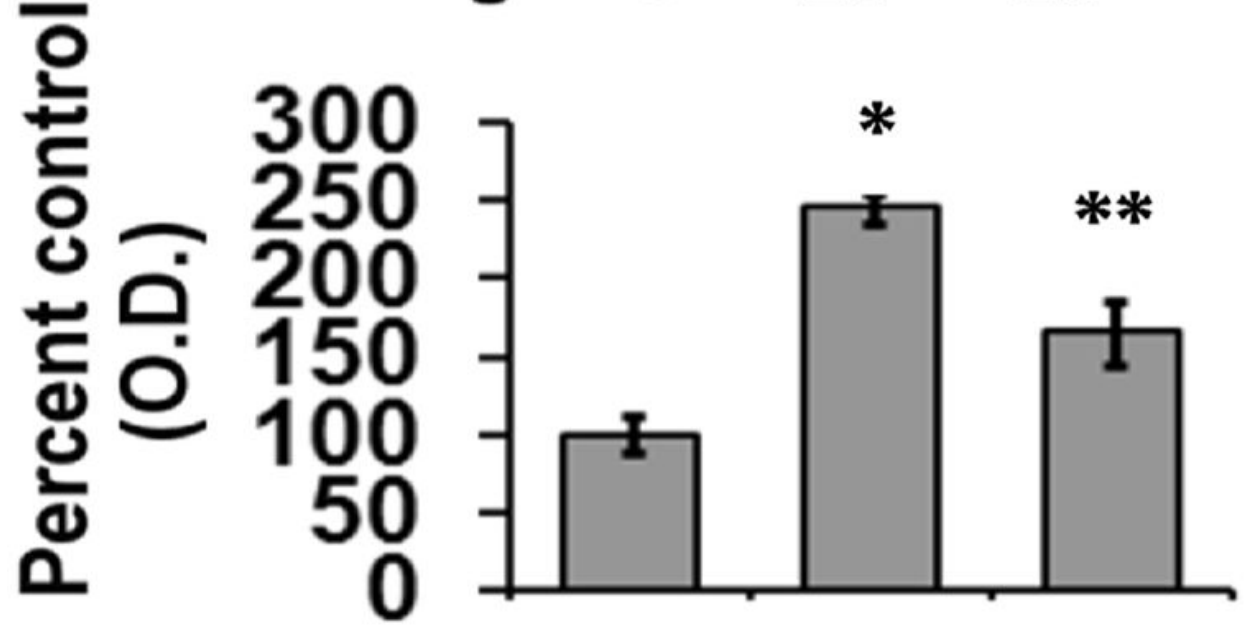

Figure 6. Effects of aging and dietary restriction on the levels of ubiquitinated protein in the heart The level of ubiquitinated protein was analyzed in the hearts of 3 month-old AL, 25 monthold AL, and 25 month-old DR rodents. A representative blot for the analyses is provided. The graph represent the mean and S.E.M. of the optical density of the immunoreactivity from 6 animals in each experimental group. *p < 0.05 compared to 3 month old AL animals; **p < 0.05 compared to 25 month old AL animals. Equal loading was confirmed by commassie staining, data not shown. 


\section{$195 k$}

$131 \mathrm{k}$

$85 k$

\section{$40 \mathrm{k}$}
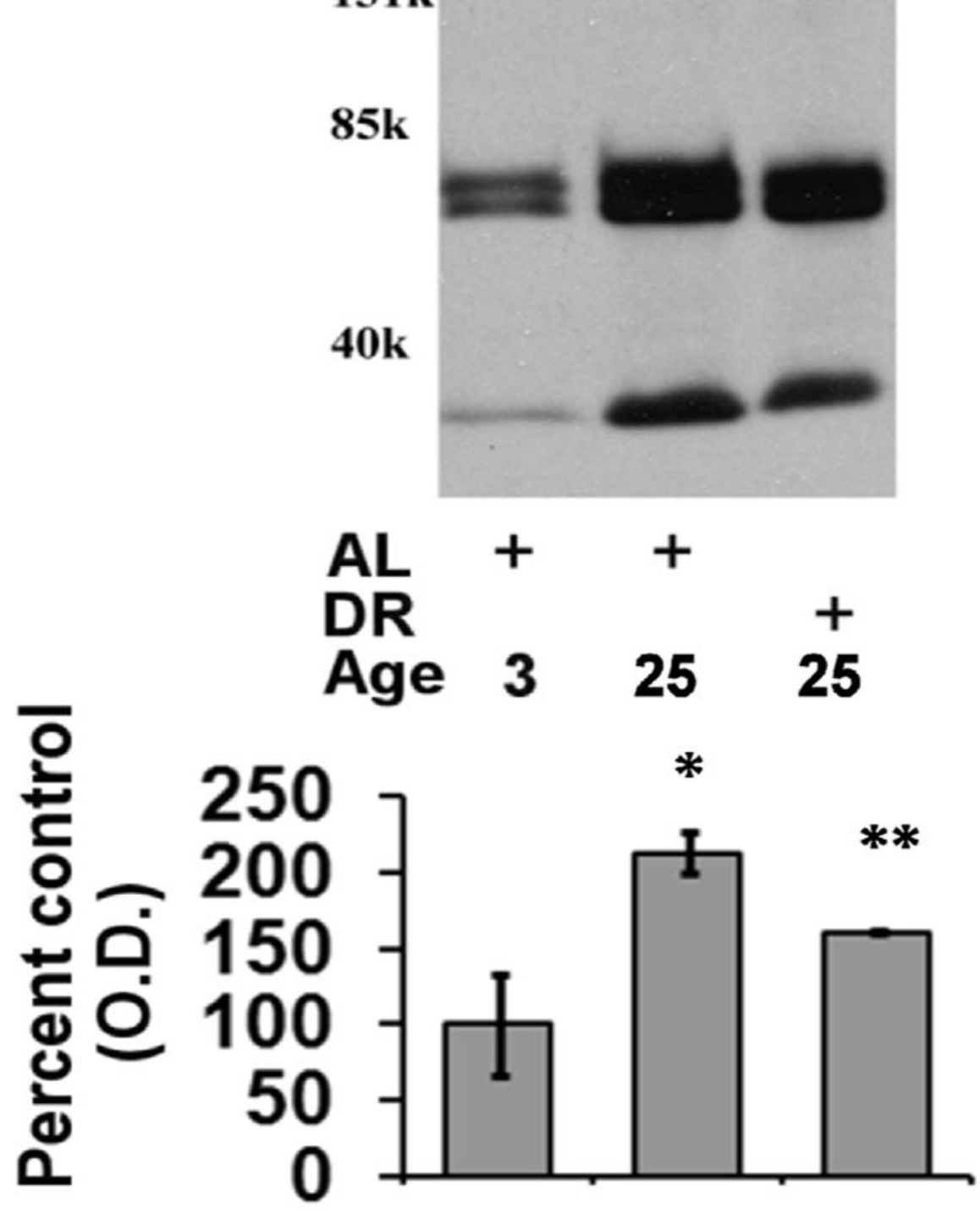

Figure 7. Effects of aging and dietary restriction on the levels of sumoylated protein in the heart The level of sumoylated protein was analyzed in the hearts of 3 month-old AL, 25 month-old AL, and 25 month-old DR rodents. A representative blot for the analyses is provided. The graphs represent the mean and S.E.M. of the optical density of the immunoreactivity from 6 animals in each experimental group. *p < 0.05 compared to 3 month old AL animals; **p < 0.05 compared to 25 month old AL animals. Equal loading was confirmed by commassie staining, data not shown. 
HNE Immunoreactivity

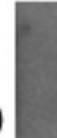

\section{0}

100

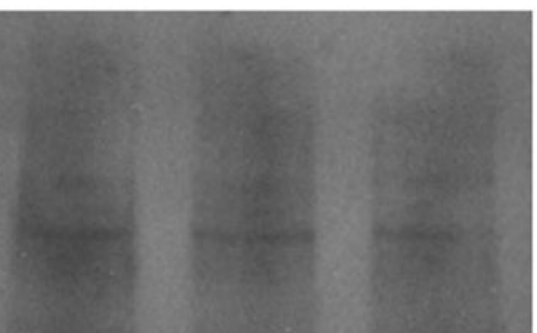

50

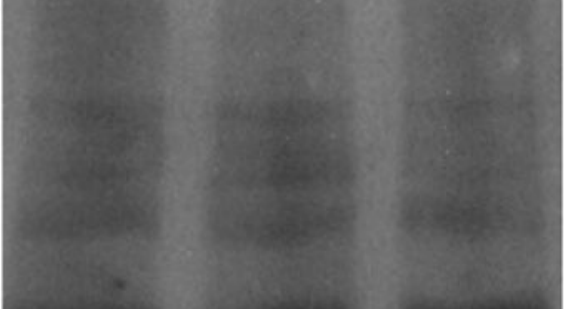

DR

AGE

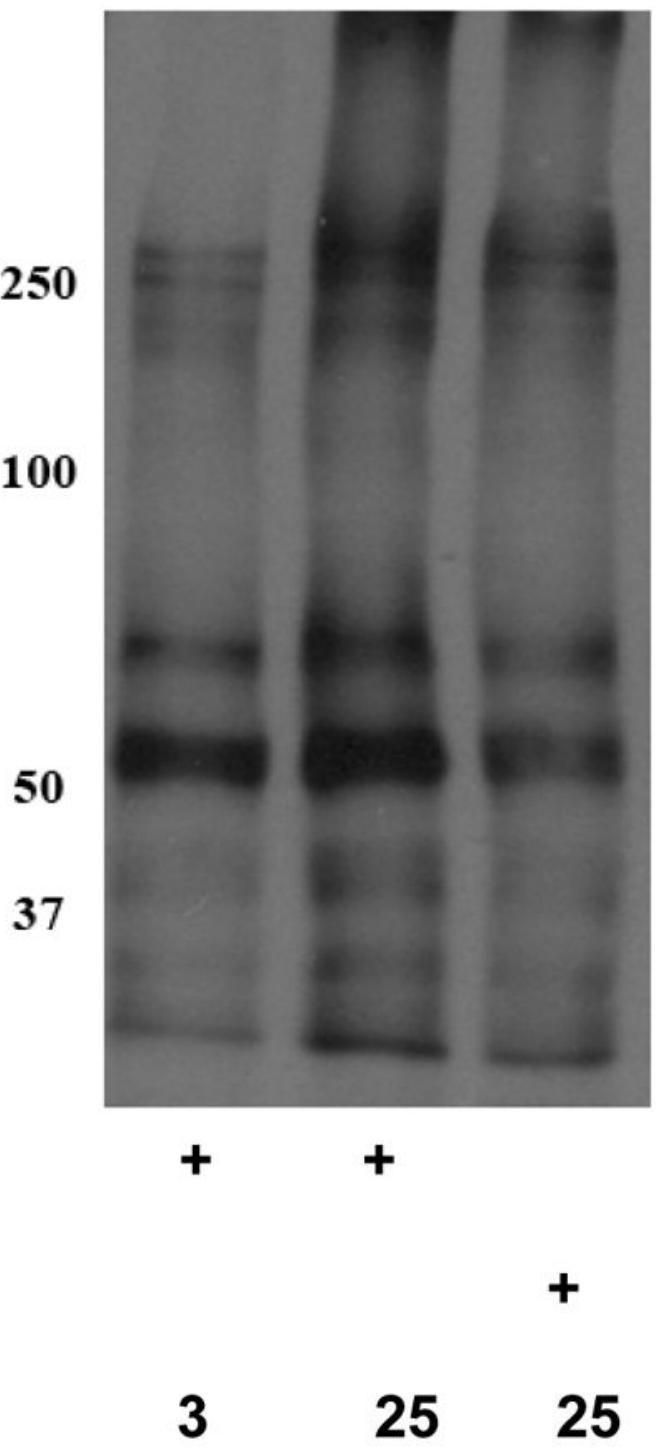

Figure 8. Effects of aging and dietary restriction on the levels of oxidized protein in the heart The level of oxidized protein was determined by analyzing the amount of HNE-modified protein and protein carbonyls present in the hearts lysates from 3 month-old AL, 25 monthold AL, and 25 month-old DR rodents. A representative blot for each analyses is provided, which represents the data obtained from 6 separate experiments. 